\title{
Coronavirus disease 2019 (COVID-19) in patients with systemic autoimmune diseases or vasculitis: radiologic presentation
}

\author{
Azadeh Eslambolchi ${ }^{1}$ - Leila Aghaghazvini ${ }^{2}$ (1) - Ali Gholamrezanezhad ${ }^{3} \cdot$ Hoda Kavosi $^{4} \cdot$ Amir Reza Radmard $^{2}$
}

Accepted: 19 September 2020 / Published online: 26 September 2020

○) Springer Science+Business Media, LLC, part of Springer Nature 2020

\begin{abstract}
Coronavirus disease 2019 (COVID-19) has transformed into a worldwide challenge, since its outbreak in December 2019. Generally, patients with underlying medical conditions are at a higher risk of complications and fatality of pneumonias. Whether patients with systemic autoimmune diseases or vasculitides, are at increased risk for serious complications associated with COVID-19, is not established yet. Computed tomography (CT) has been employed as a diagnostic tool in the evaluation of patients with clinical suspicion of severe acute respiratory syndrome coronavirus 2 (SARS-Cov-2) infection with a reported sensitivity of higher than reverse transcription polymerase chain reaction (RT-PCR) test. Multifocal bilateral ground-glass opacities (GGOs) with peripheral and posterior distribution and subsequent superimposition of consolidations are considered the main imaging features of the disease in chest CT. However, chest CT images of underlying rheumatologic or autoimmune diseases or vasculitides, such as systemic sclerosis, systemic lupus erythematosus, rheumatoid arthritis, Behçet disease, and granulomatosis with polyangiitis, especially those with extensive lung involvement can overshadow or obliterate features of COVID-19. In addition, CT findings of such diseases may resemble manifestations of COVID-19 (such as ground glass opacities with or without superimposed consolidation), making the diagnosis of viral infections, more challenging on imaging. Comparing the imaging findings with prior studies (if available) for any interval change is the most helpful approach. Otherwise, the diagnosis of COVID-19 in such patients must be cautiously made according to the clinical context and laboratory results, considering a very high clinical index of suspicion on imaging.
\end{abstract}

Keywords COVID-19 $\cdot$ Pneumonia $\cdot$ Vasculitis $\cdot$ Granulomatosis polyangiitis $\cdot$ CT scan

\section{Highlights}

Azadeh Eslambolchi and Leila Aghaghazvini have contributed equally to the work.

\section{Leila Aghaghazvini}

aghaghazvini.leila@gmail.com

Azadeh Eslambolchi

azadeh.eslambolchi@gmail.com

Ali Gholamrezanezhad

Ali.Gholamrezanezhad@med.usc.edu

Independent Researcher, Seattle, USA

2 Department of Radiology, Shariati Hospital, Tehran University of Medical Sciences (TUMS), Jalal-e-Al-e-Ahmad Hwy, Tehran, Iran

3 Department of Radiology, Keck School of Medicine, University of Southern California (USC), Los Angeles, CA, USA

4 Rheumatology Research Center, Shariati Hospital, Tehran University of Medical Sciences, Tehran, Iran
- The risk of COVID-19 and its associated complications is considered to be increased in patients with preexisting medical conditions such as rheumatic autoimmune disease or vasculitis.

- Chest CT with about $97 \%$ sensitivity has been extensively employed in the diagnosis and follow-up of patients with COVID-19, while RT-PCR with high specificity is the confirmative test. Chest $\mathrm{CT}$ has been playing a key role in many healthcare settings due to the inadequacy of laboratory kits for diagnosis and more availability of CT.

- Chest CT images of underlying rheumatologic conditions with pulmonary involvement, such as systemic sclerosis, systemic lupus erythematosus, rheumatoid arthritis, Behçet disease, and granulomatosis with polyangiitis, can obliterate or resemble features of COVID-19. 
- Diagnosis of COVID-19 in patients with underlying rheumatic autoimmune disease or vasculitis should be cautiously made according to the clinical context and RT-PCR results, considering a high clinical index of suspicion on imaging.

- We discussed chest CT features in a number of patients with COVID-19 and underlying rheumatic diseases or vasculitis. There is a paucity of similar studies in the literature.

\section{Introduction}

Infection with severe acute respiratory syndrome coronavirus 2 (SARS-Cov-2) has transformed into a worldwide challenge, since its outbreak in December 2019. By March 11, World Health Organization (WHO) characterized coronavirus disease 2019 (COVID-19) as a pandemic [1], and as of July 21, over 14,500,000 confirmed cases have been recorded globally, claiming more than 607,000 lives [2]. COVID-19 presents with a range of clinical symptoms, including, but not limited to, dyspnea, cough, and constitutional symptoms. The intensity of symptoms and clinical manifestation of the disease varies from mild to severe, contingent on several factors, including but not limited to age, comorbidities, and integrity of the immune system as well as virological factors [3-6]. Like other chronic diseases, such as coronary heart disease, diabetes mellitus, hypertension, malignancies, chronic lung disease, asthma, human immunodeficiency virus (HIV) infection, liver disease, and chronic kidney disease [7], patients with rheumatic diseases are considered to be at an increased risk of respiratory infections and their complications $[8,9]$. This may be due to disability and immune dysregulation. Immune modulating treatments and steroids also predispose patients to infection [10,11]; however, some articles are against increased infection rate, higher risk of acute respiratory distress syndrome (ARDS), or other life threatening complications related to COVID-19 in rheumatologic patients $[12,13]$.

In a more severe type of COVID-19, uncontrolled and overwhelming release of cytokines, known as cytokine release syndrome (CRS) can lead to many pathological processes, suggesting that a severe immune reaction in the body can also be accountable for tissue damage, in addition to viral virulence. Cytokine release syndrome can also be induced by macrophage activation in patients with immune-related diseases and can be responsible for the immune pathogenesis of many pathological processes in these patients. Based on the similarity of the underlying pathogenesis of cytokine release in patients with COVID-19 and rheumatologic diseases, some overlap in imaging findings of these patients can be expected [14].
Clinical presentation, exposure history, reverse transcription polymerase chain reaction (RT-PCR) testing, and computed tomography (CT), each has their own diagnostic role in COVID-19, especially in the early stages of the disease [15]. Chest CT with about $97 \%$ sensitivity has been extensively employed in the diagnosis and follow-up of patients with COVID-19, while RT-PCR with high specificity is the confirmative test for the diagnosis. Although some scientific advisors do not agree with the role of chest $\mathrm{CT}$ as a routine screening modality in this setting, it has been playing a key role in many healthcare settings due to the inadequacy of laboratory kits for diagnosis and more availability of CT [16-19]. More recently, with the wide availability of RTPCR kits, CT is mainly used for the evaluation of COVID-19 complications and suspect alternative diagnoses [20].

Chest CT findings in COVID-19 has been extensively discussed in literature. Multifocal bilateral ground-glass opacities (GGOs) with a peripheral and posterior distribution and later superimposition of consolidations are considered as the dominant radiologic picture of the disease [21, 22]. Less common findings of SARS-CoV-2 infection in CT include, CT halo sign, interlobular septal thickening, crazy paving pattern, bronchiectasis, pleural effusion, pericardial effusion, mediastinal lymphadenopathy [15, 23, 24], airway changes, reversed halo, vascular change as increased mural thickness or dilatation in involved region of parenchyma, and focal pleural thickening [25, 26]. In spite of numerous studies attempting to draw the map of CT characteristics in COVID-19, articles studying image characteristics in the patients with preexisting rheumatologic disease are of great scarcity [19].

Chest CT images of underlying rheumatologic conditions, especially those with pulmonary involvement, can overshadow or obliterate features of COVID-19, especially in the early stages of the infection. Radiologic features of lung involvement in rheumatic immune disease or vasculitis (usually as nonspecific interstitial pneumonitis-NSIP) can also simulate CT manifestations of COVID-19, considering numerous potential atypical manifestations of COVID-19. To address these issues, as an IRB approved retrospective cases series, we go over a number of cases of our tertiary hospital system with serologically confirmed COVID-19 pneumonia and underlying rheumatologic conditions or vasculitis and discuss their imaging picture. As the case series was performed retrospectively, the institutional review board waived informed consent.

\section{Rheumatoid arthritis}

While immunosuppressive medications, comorbidities, and immune dysregulation, in patients suffering from rheumatologic diseases lead to an increased risk of infection [10], 
there is still uncertainty about the increased rate of COVID19 and greater risk of complications in patients with rheumatoid arthritis (RA) [12]. Furthermore, the increasing knowledge about the pathological process of SARS-CoV-2 infection has resulted in some anti-rheumatoid medications such as Chloroquine and Hydroxychloroquine to be considered as a potentially effective option in the management of COVID-19 pneumonia [11].

Pulmonary involvement is a frequent extra-articular feature in RA. Among the radiologic findings of RA-related lung disease, GGOs and reticulations are the most common CT features. Other pulmonary findings include lung nodules, bronchiectasis, bronchiolitis, and those related to interstitial involvement, such as nonspecific interstitial pneumonia (NSIP), usual interstitial pneumonia (UIP), and organizing pneumonia. Pericardial and pleural effusion are also frequently seen $[27,28]$.

Figure 1 demonstrates the chest CT images of a known case of rheumatoid arthritis with typical clinical symptoms and serological confirmation of COVID-19. The resemblance of CT findings in acute COVID-19 pneumonia and RA-related pulmonary disease indicates that diagnosis of COVID-19 in this group of patients should be cautiously made based on clinical context and mainly serologic data. In such cases, comparing the recent $\mathrm{CT}$ with any prior imaging, if available, can be most beneficial.

\section{Systemic lupus erythematosus}

While some articles argue against the increased risk of infection and a higher rate of ARDS or other severe complications associated to COVID-19 in patients suffering from systemic lupus erythematosus (SLE) [29], other studies are in favor of a more severe and complicated course of COVID-19 in this setting [30]. Further studies are needed to clarify the effect of SLE in the morbidity and mortality of COVID-19.

Pulmonary parenchyma is frequently involved in the course of SLE. Lung disease in SLE can be a notable cause of morbidity and mortality [31]. In a study by Li et al. the most common manifestations of thoracic involvement in SLE were interstitial pulmonary changes (including GGOs, honeycombing, subpleural lines, interstitial septal thickening, and emphysema) (66.67\%), pleural disease $(61.54 \%)$, parenchymal changes (including consolidation and patchy infiltration) $(25.64 \%)$ and vascular changes (12.82\%) [32]. Due to potential resemblance of presentations of COVID-19 pneumonia in chest
Fig. 1 A 40-year-old woman with a history of rheumatoid arthritis for over 5 years presented with fever, dry cough, shortness of breath, and fatigue. Reverse transcription polymerase chain reaction was positive for SARS-CoV-2 infection. Axial chest $\mathrm{CT}$ a-c shows bilateral multifocal GGOs (arrows; a-c) and early consolidation in right middle lobe (dotted arrow; c), likely due to the patient's known COVID-19. There is peribronchovascular thickening (arrowhead; a) and some subpleural reticulation (thin arrow; c) suggestive of interstitial pulmonary involvement secondary to underlying rheumatoid arthritis versus early superimposition of consolidation due to COVID-19. Differentiation is difficult and may need follow-up post-treatment imaging or comparison with any prior (pre-COVID-19) imaging, if available
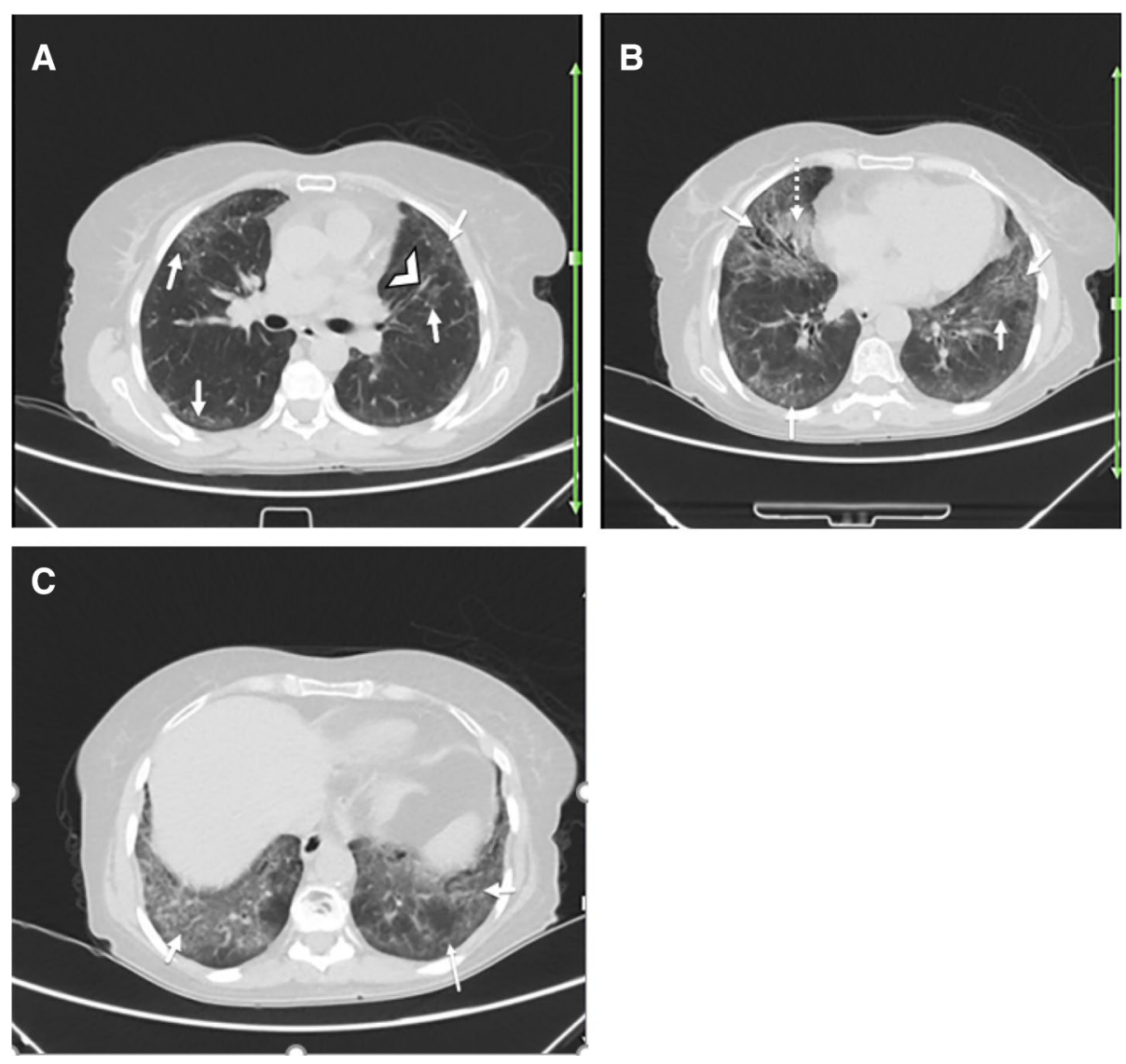
CT, with features of pulmonary involvement in SLE, differentiating these two entities can be challenging.

Figures 2 and 3 demonstrate the chest CT images of two known cases of SLE with clinical features and serological confirmation of COVID-19 pneumonia. Although Fig. 2 shows an atypical presentation of COVID-19 on imaging, Fig. 3 demonstrates a more typical imaging manifestation of the disease. Pleural thickening in Fig. 2 is more likely suggestive of SLE-related disease; however, pleural effusion and thickening can be infrequently seen in COVID-19 pneumonia.

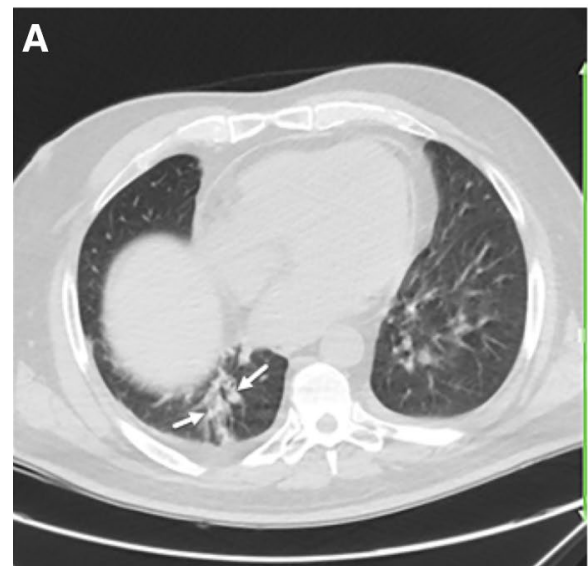

Fig. 2 A 25-year-old woman with a history of systemic lupus erythematosus for over 4 years, presented with 3 days of fever, myalgia, and dry cough. Reverse transcription polymerase chain reaction was positive for SARS-CoV-2. Axial chest CT a, b shows patchy airspace opacity (early consolidation versus atelectasis) (arrows; a) in the

\section{Systemic sclerosis}

Lung parenchyma is among the most important visceral organs that can be affected by the fibrotic process of collagen accumulation in systemic sclerosis (SSc). Pulmonary hypertension and Interstitial Lung Disease (ILD) are common manifestations of SSc and develop in near 75\% of patients [33]. Patients with preexisting SSc, infected with SARA$\mathrm{CoV}-2$, can be at higher risk for fatal pulmonary complications, comparing to other autoimmune diseases, because of higher prevalence of pulmonary disease and common use of immune suppressive treatments [34].

Involvement of the heart in patients with SSc, due to primary cardiomyopathy or secondary to pulmonary

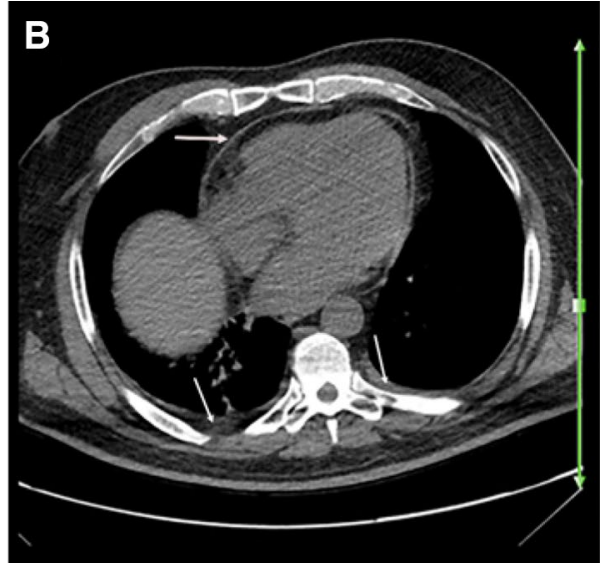

right lower lobe, which may or may not represent the patient's known COVID-19 pneumonia. Presence of pleural and pericardial thickening (arrows; b) is mostly suggestive of pleuropericardial disease due to underlying SLE
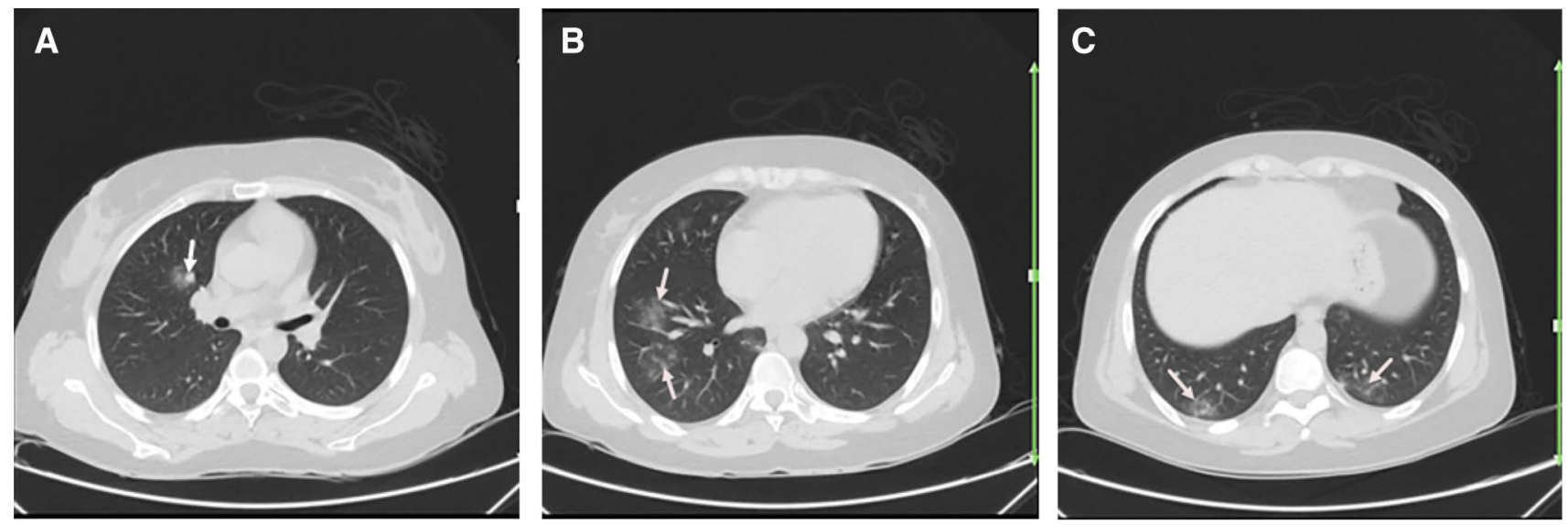

Fig. 3 A 30-year-old woman with a history of well-controlled SLE, presented with dyspnea, dry cough, and fever. Reverse transcription polymerase chain reaction was positive for SARS-CoV-2. Axial chest
CT a-c shows bilateral multifocal GGO (arrows; a-c), consistent with the patient's known COVID-19 pneumonia 
hypertension, is associated with an increased rate of morbidity and mortality. Moreover, according to WHO, patients with underlying cardiovascular disease have increased susceptibility to SARS-CoV-2 infection and are prone to more severe disease with worse prognosis [32]. These facts call for more attention to dealing with SSc patients with respect to SARS-CoV-2 infection. In our search, there were only few articles dealing with COVID-19 in patients suffering from SSc and ILD, with only one providing chest CT information $[35,36]$.

Classically, SSc affects posterior, subpleural, and basilar portions of lungs, initially with subtle GGOs and reticular interstitial thickening in chest CT, which makes it very difficult to differentiate it from COVID-19. Many reported cases of COVID-19 pneumonia have resembled nonspecific interstitial pneumonitis (NSIP), as both tend to have peripheral and basilar ground glass opacities [24, 34]. However, in advanced stages of SSc, changes of scleroderma interstitial lung disease (SILD) may appear in CT, manifesting as fibrotic changes with decreased lungs volume, honeycomb cystic changes, traction bronchiectasis, and bronchiolectasis [37].

Figures 4 and 5 demonstrate the chest CT images of two known cases of systemic sclerosis and ILD with clinical manifestations, in favor of COVID-19 pneumonia. In the first case, chest CT findings of subpleural reticulation mostly in posterior segments of the lungs, traction bronchiectasis, and peribronchovascular thickening are mostly consistent with ILD in SSc. Bilateral patchy GGOs are nonspecific in this setting and may represent superimposed acute COVID-19 pneumonia versus secondary to the patient's known inflammatory disease with possible pulmonary involvement. In the second case, there are some subpleural reticulation mostly consistent with interstitial involvement in SSc and sizeable bilateral pleural and pericardial effusion. Based on current literature, critically ill COVID-19 patients with preexisting comorbidities show a higher incidence of pericardial and pleural effusion, compared to the general population [38].

\section{Behçet disease}

Behçet disease is a rare multisystem and inflammatory disorder with an unknown cause. It is more common in young men, and in the Mediterranean descent. Uveitis and recurring genital and mouth ulcers are the typical triad of this disease. Joints, central nervous system, gastrointestinal system, pulmonary parenchyma, and cardiovascular system can also be affected in Behçet disease [39]. Both veins and arteries can be affected in the form of vasculitis, though veins are involved more frequently. Thoracic vascular involvement is
Fig. 4 A 62-year-old woman with a history of known scleroderma for over 6 years, presented with fever, dry cough, fatigue, and dyspnea of 3 days duration. Reverse transcription polymerase chain reaction was positive for SARS-CoV-2. Axial chest CT shows bilateral multifocal patchy GGOs, predominantly involving the left lung (thick arrows; $\mathbf{a}-\mathbf{c}$ ) consistent with COVID-19 pneumonia. There are subpleural curvilinear and peribronchovascular reticulation and interstitial fibrosis (thin arrows; $\mathbf{b}, \mathbf{c}$ suggestive of interstitial lung disease due to scleroderma. Evidence of esophageal dilatation (dotted arrow; a) and cardiomegaly (dotted arrow; d) may be suggestive of scleroderma related esophageal and cardiac involvement, respectively
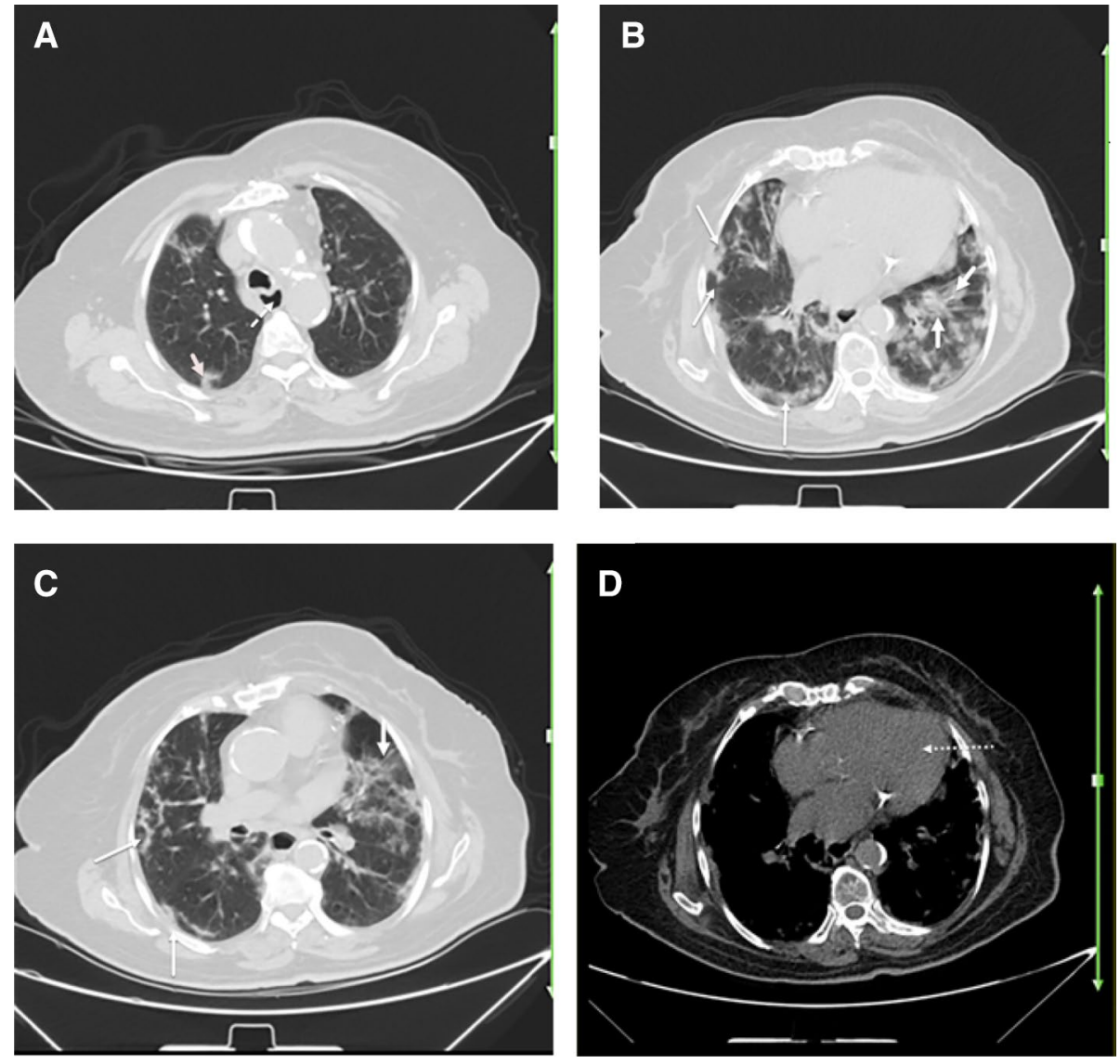

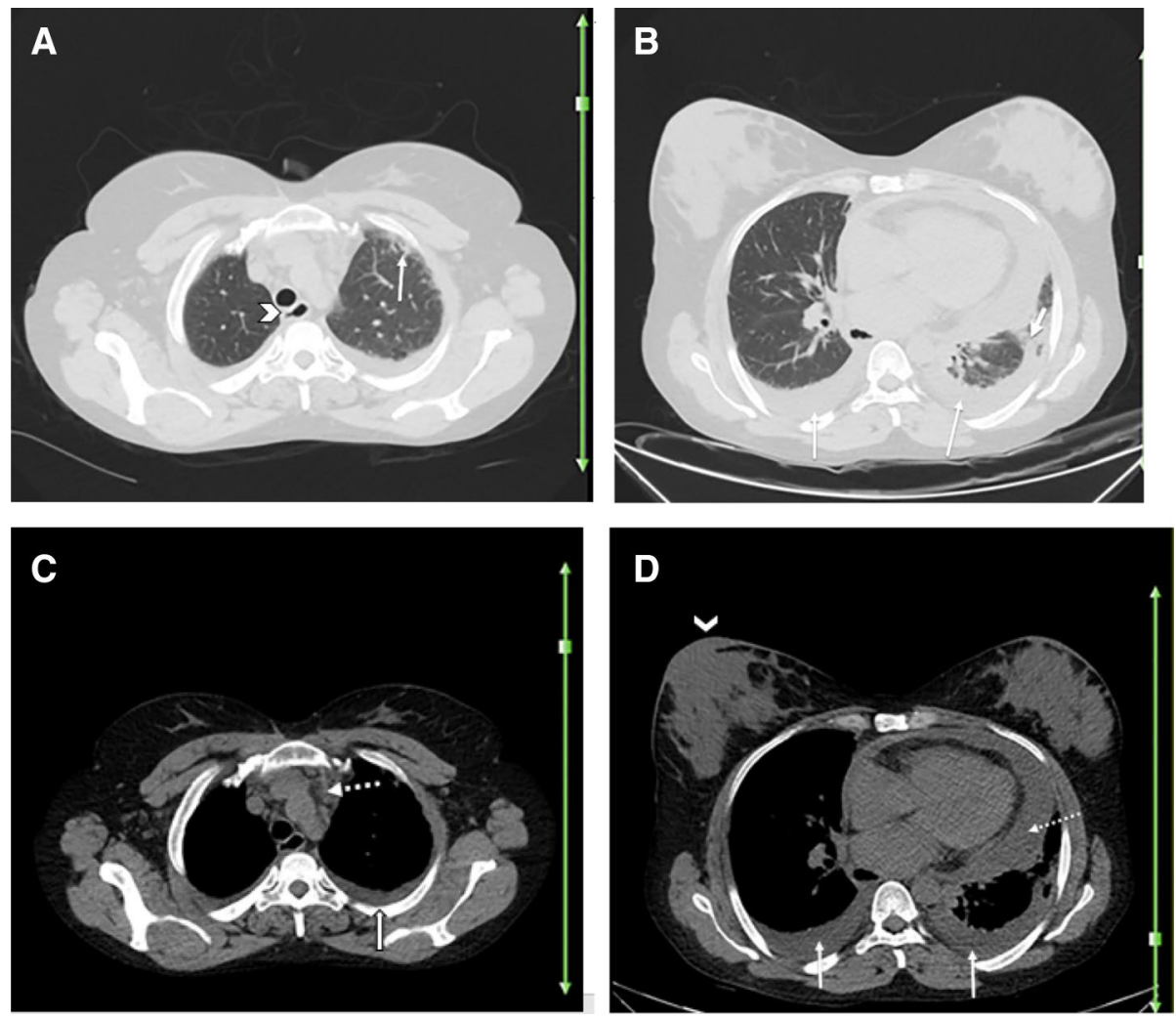

Fig. 5 A 49-year-old woman with a history of well-controlled scleroderma and uncomplicated pregnancy and delivery 3 months ago, presented with fever, dry cough, and myalgia for 2 days. $\mathrm{O}_{2}$ saturation was $81 \%$. Reverse transcription polymerase chain reaction was negative for SARS-CoV-2. Axial chest CT a-d shows bilateral pleural effusion (thin arrows; b-d) and pericardial effusion (dotted arrow; d) which are uncommon findings of COVID-19 in general population, but can be more common in patients with preexisting disease. Small focal patchy GGO in the left lower lobe (thick arrow; b) is noted, which can be related to COVID-19 or secondary to compressive atelectasis. Although the RT-PCR test was negative in this patient, due to high clinical suspicion, living in an epidemic region, suboptimal

the most frequent cause of mortality. It happens in about $25-30 \%$ of cases and can display as pulmonary artery embolism, aneurysm of pulmonary artery, or thrombosis of vena cava [40]. Pleura and lung parenchyma are involved in $1-10 \%$ of cases. Pulmonary artery thrombosis and occlusion, because of vasculitis, may lead to hemorrhage and infarction. Parenchymal disease can manifest as pulmonary nodules, bronchiectasis, atelectasis, and interstitial lung disease. Less common manifestations of pulmonary involvement are fibrotic bands, emphysema, eosinophilic pneumonia, bronchitis, and small airway disease [39].

Behçet disease has been included in different classification systems ranging from vasculitis to rheumatic autoimmune, and spondyloarthropathies. Although few patients with vasculitis [41] or spondyloarthritis [42], infected with SARS-CoV-2 have been reported sporadically, we have sensitivity of serologic test and recuperation after 2 weeks, COVID19 was the clinical diagnosis for this patient. Subpleural interstitial reticulation in left upper lobe is suggestive of scleroderma-related lung involvement (thin arrow; a). A soft tissue opacity in left anterior mediastinum (dotted arrow; c) is noted, suggestive of thymus hyperplasia, which can be related to underlying scleroderma. Evidence of esophageal dilatation (arrowhead; a) may be suggestive of scleroderma related esophagus involvement and warrants further evaluation. Cutaneous thickening of the right breast (arrowhead; d) is noted, which can be due to infectious/inflammatory diseases of lactation phase versus SSc-related skin change or neoplastic in etiology and warrants correlation with physical exam findings

not found any published report, dealing with COVID-19 in Behçet disease.

Figure 6 demonstrates the chest CT images of a known case of Behçet disease with clinical findings in favor of COVID-19 and laboratory-confirmed COVID-19 pneumonia. A patch of GGO is noted in the left upper lobe, consistent with COVID-19 pneumonia. There is a nodular density in central lingula, which can be suggestive of nodular involvement of pulmonary parenchyma in Behçet disease. As mentioned before, nodular opacity can be a sign of vasculitis and parenchymal involvement in Behçet disease. However, interpretation of COVID-19 CT findings in Behçet disease, like other multisystem chronic disorders, should be made in the setting of clinical context, lab results, with considering possible differential diagnosis. 

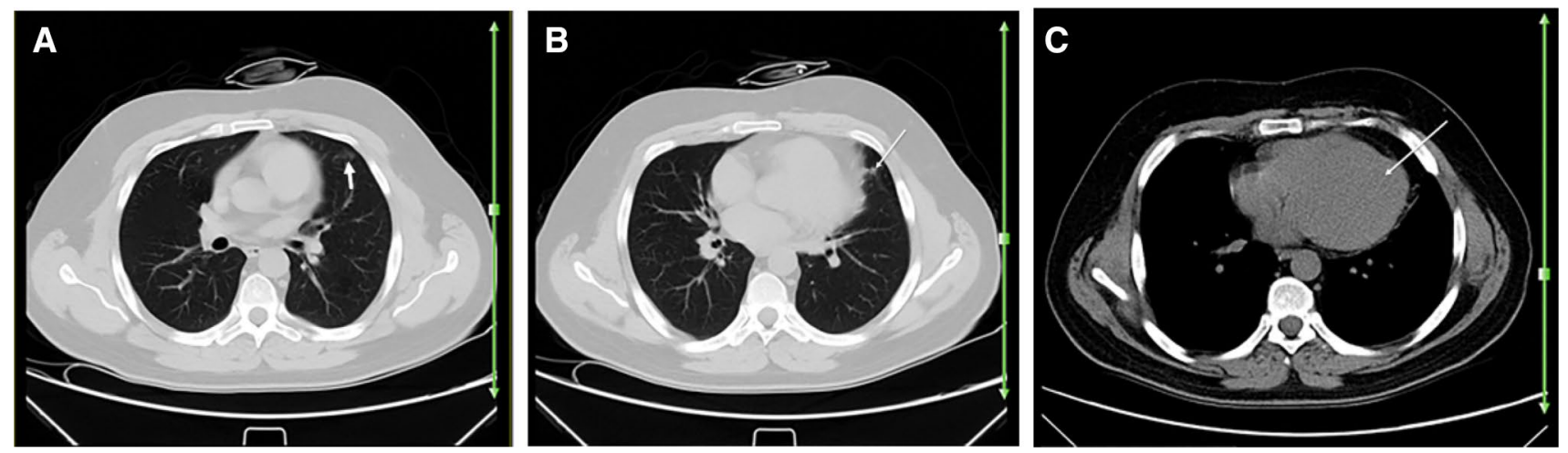

Fig. 6 A 35-year-old man with a known history of Behçet disease for 6 years, presented with fever and dyspnea. Reverse transcription polymerase chain reaction was positive for SARS-CoV-2. Axial chest CT a-c shows a faint patchy GGO in anterior segment of left upper lobe (arrow; a), which can be due to known SARS-Cov-2 infection or sec-

\section{Granulomatosis with polyangiitis (wegener's granolumatosis)}

Granulomatosis with polyangiitis (GPA), is an uncommon necrotizing vasculitis that was previously known as Wegener's granulomatosis. It presents with a clinical and pathological manifestations of upper airway, pulmonary, vascular, and renal system involvement. Many organs in the body can be involved; however, thoracic involvement in the course of the disease is the main problem. Poor or well-defined nodules of varying size, GGOs, consolidations, masses, and cavities are among common radiologic manifestations. Trachea and bronchial structures are also frequently involved. Tracheal involvement is usually seen as focal wall thickening or segmental stenosis. Distal airway involvement can present with bronchial wall thickening and bronchiectasis and may lead to pneumonia and atelectasis [43].

In Wegener's granulomatosis, pleura, mediastinum, and heart are not affected commonly; however, pleural involvement most frequently manifests as pleural effusion. This can be due to affected pleura itself in the course of GPA or secondary to renal involvement. In up to 50\% of lung nodules, cavitation can occur, especially in larger nodules with sizes over $2 \mathrm{~cm}$. Hemorrhage in surrounding tissue of a nodule or cavity, can cause "halo sign" or "atoll sign" (reverse halo), respectively. Widespread ground-glass opacity and consolidation are also common findings in patients with GPA. Bilateral perihilar or peribronchovascular distribution is the most common pattern of GGOs. Ground-glass opacities and consolidations can disappear by time, similar to pulmonary nodules, regardless of therapy [43, 44].

Although our GPA patient in Fig. 7 demonstrates some nonspecific features that can be attributed to either COVID19 or underlying autoimmune inflammatory disease, he also demonstrates typical findings of sino-nasal involvement in ondary to pulmonary involvement of Behchet. Small nodular opacity involving the paracardiac lingula is noted (arrow; b), most likely suggestive of parenchymal nodular disease of Behçet, as nodular disease is an uncommon manifestation of COVID-19. In mediastinal window, evidence of mild cardiomegaly is noticeable (arrow; c)

GPA, as an erosive osseous defect of nasal septum due to necrotizing vasculitis and signs of sinusitis. Nasal cavity and paranasal sinuses are involved in the course of GPA frequently. Due to decreased blood flow in the setting of vasculitis, tissue damage and nasal bone destruction can happen [45]. Due to resemblance of common pattern of pulmonary involvement in Granulomatosis with polyangiitis with typical findings of COVID-19 pneumonia, interpretation of chest $\mathrm{CT}$ of these patients should be made with special attention to distinctive patterns of each disease, consideration of clinical context, and emphasis on RT-PCR.

\section{Conclusion}

The risk of COVID-19 and its associated complications are increased in patients with preexisting medical conditions. Patients with rheumatologic diseases and vasculitis can be at an increased risk of infection, due to underlying impairment of immunity and adverse effects of corticosteroids or other immunosuppressive therapies on the immune system. Some imaging features of pulmonary involvement in these patients can mimic COVID-19. CT images of underlying morbidities may overshadow or obliterate features of COVID-19, especially in the early stages of the infection. Therefore, differentiation of superimposed infection with SARS-Cov-2 from underlying autoimmune pulmonary disease can be challenging, and the interpretation should be made in the context of serological data and clinical setting. Correlation of the imaging finding with those of the prior studies is most useful, if available.

However in majority of rheumatologic conditions, such as RA and SLE, there is no indication for pulmonary screening in asymptomatic patients. In these cases (like our reported patients), usually there is no prior/baseline 

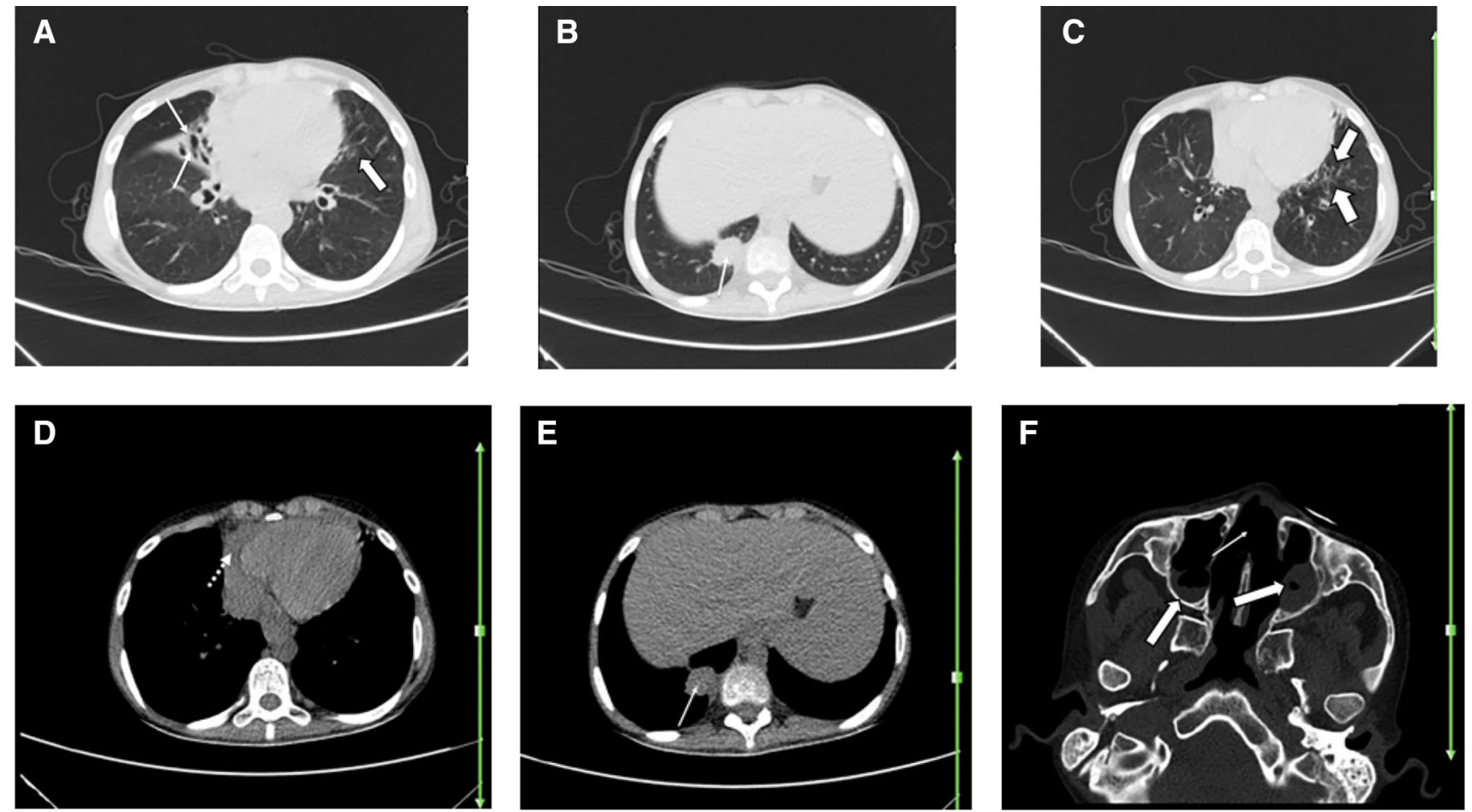

Fig. 7 A 22-year-old man with a known history of granulomatosis with polyangiitis for over 1 year, presented with fever, dry cough, and shortness of breath. Reverse transcription polymerase chain reaction was positive for SARS-CoV-2. Axial chest CT shows ground glass opacities in lingula (thick arrows; a, c), which can be due to the patient's known COVID-19 pneumonia versus pulmonary involvement by the underlying autoimmune disease. Diffuse central bronchiectasis (thin arrow; a), subpleural soft tissue density, non-calcified mass of $35 \times 25 \mathrm{~mm}$ in the paravertebral basal segment of right lower

imaging for comparison and the diagnosis should mainly rely upon clinical and serological data. In some other rheumatologic conditions, that the primary pulmonary involvement resembles NSIP (such as scleroderma) or patchy ground glass appearance due to alveolar hemorrhage in Granulomatosis with Polyangiitis, sometimes differentiation is confusing. Therefore, differentiation of superimposed infection with SARS-Cov-2 from underlying autoimmune pulmonary disease can be challenging, and the interpretation should be made in the context of serological data and clinical setting. Correlation of the imaging finding with those of the prior studies is most useful, if available.

Follow-up imaging is another useful tool to differentiate pulmonary infiltrations of rheumatologic disorders from infectious opacities of coronavirus pneumonia, as the former has a slow progression without appropriate therapy, while the latter (like most other infectious pneumonias) is more rapidly progressing or regressing. On the other hand, follow-up imaging to complete resolution of the inflammatory infiltrations is recommended, as this population is prone to lobe (arrows; b, e), pericardial effusion (dotted arrow; d), nasal septal osseous defect (thin arrow; f), and suggestive signs of sinusitis such as soft tissue opacity in left maxillary sinus, as well as fluid level in right maxillary sinus (thick arrows; f) are noted. These findings are suggestive of involvement of lower airways, pulmonary parenchyma, pericardial, and sino-nasal structures related to granulomatosis with polyangiitis. However, pericardial effusion can also be a rare finding in COVID-19

long-term consequences of the coronavirus infection and residual pulmonary scarring $[46,47]$.

Funding None.

\section{Compliance with ethical standards}

Conflict of interest The authors declare that they have no conflict of interest.

\section{References}

1. WHO Timeline-COVID-19 (2020) Who.int. 2020. https://www. who.int/news-room/detail/27-04-2020-who-timeline---covid-19. Accessed 21 July 2020

2. COVID-19 situation reports (2020) Who.int. 2020. https://www. who.int/emergencies/diseases/novel-coronavirus-2019/situationreports. Accessed 21 July 2020

3. Huang C, Wang Y, Li X, Ren L, Zhao J, Hu Y, Zhang L, Fan G, Xu J, Gu X, Cheng Z, Yu T, Xia J, Wei Y, Wu W, Xie X, Yin W, Li H, Liu M, Xiao Y, Gao H, Guo L, Xie J, Wang G, 
Jiang R, Gao Z, Jin Q, Wang J, Cao B (2020) Clinical features of patients infected with 2019 novel coronavirus in Wuhan, China. Lancet 395(10223):497-506. https://doi.org/10.1016/S0140 -6736(20)30183-5. Erratum in: Lancet

4. Liu K, Fang YY, Deng Y et al (2020) Clinical characteristics of novel coronavirus cases in tertiary hospitals in Hubei Province. Chin Med J (Engl) 133(9):1025-1031. https://doi.org/10.1097/ CM9.0000000000000744

5. Du RH, Liang LR, Yang CQ, Wang W, Cao TZ, Li M, Guo GY, Du J, Zheng CL, Zhu Q, Hu M, Li XY, Peng P, Shi HZ (2020) Predictors of mortality for patients with COVID-19 pneumonia caused by SARS-CoV-2: a prospective cohort study. Eur Respir J 55(5):2000524. https://doi.org/10.1183/13993003.00524-2020

6. CDC COVID-19 Response Team (2020) Severe outcomes among patients with coronavirus disease 2019 (COVID-19)-United States, February 12-March 16, 2020. MMWR Morb Mortal Wkly Rep 69(12):343-346. https://doi.org/10.15585/mmwr.mm6912e2

7. Zhou F, Yu T, Du R, Fan G, Liu Y, Liu Z, Xiang J, Wang Y, Song B, Gu X, Guan L, Wei Y, Li H, Wu X, Xu J, Tu S, Zhang Y, Chen H, Cao B (2020) Clinical course and risk factors for mortality of adult inpatients with COVID-19 in Wuhan, China: a retrospective cohort study. Lancet 395(10229):1054-1062. https ://doi.org/10.1016/S0140-6736(20)30566-3. Erratum in: Lancet

8. Atzeni F, Masala IF, di Franco M, Sarzi-Puttini P (2017) Infections in rheumatoid arthritis. Curr Opin Rheumatol 29(4):323330. https://doi.org/10.1097/BOR.0000000000000389

9. Hsu CY, Ko CH, Wang JL, Hsu TC, Lin CY (2019) Comparing the burdens of opportunistic infections among patients with systemic rheumatic diseases: a nationally representative cohort study. Arthritis Res Ther 21(1):211. https://doi.org/10.1186/s1307 5-019-1997-5

10. Mehta B, Pedro S, Ozen G, Kalil A, Wolfe F, Mikuls T, Michaud K (2019) Serious infection risk in rheumatoid arthritis compared with non-inflammatory rheumatic and musculoskeletal diseases: a US national cohort study. RMD Open 5(1):e000935. https://doi. org/10.1136/rmdopen-2019-000935

11. Favalli E, Ingegnoli F, De Lucia O et al (2020) COVID-19 infection and rheumatoid arthritis: faraway, so close! Autoimmun Rev 19(5):102523. https://doi.org/10.1016/j.autrev.2020.102523

12. Horisberger A, Moi L, Ribi C, Comte D (2020) Impact of COVID19 pandemic on SLE: beyond the risk of infection. Lupus Sci Med 7(1):e000408. https://doi.org/10.1136/lupus-2020-000408

13. Liu B, Li M, Zhou Z, Guan X, Xiang Y (2020) Can we use interleukin-6 (IL-6) blockade for coronavirus disease 2019 (COVID19)-induced cytokine release syndrome (CRS)? J Autoimmun. https://doi.org/10.1016/j.jaut.2020.102452

14. Coronavirus Disease 2019 (COVID-19) (2020) Centers for Disease Control and Prevention. https://www.cdc.gov/coronaviru s/2019-ncov/hcp/clinical-criteria.html. Accessed 21 May 2020

15. Salehi S, Abedi A, Balakrishnan S, Gholamrezanezhad A (2020) Coronavirus disease 2019 (COVID-19) imaging reporting and data system (COVID-RADS) and common lexicon: a proposal based on the imaging data of 37 studies. Eur Radiol. https://doi. org/10.1007/s00330-020-06863-0

16. Huang EP, Sung CW, Chen CH, Fan CY, Lai PC, Huang YT (2020) Can computed tomography be a primary tool for COVID19 detection? Evidence appraisal through meta-analysis. Crit Care 24(1):193. https://doi.org/10.1186/s13054-020-02908-4

17. Ai T, Yang Z, Hou H, Zhan C, Chen C, Lv W, Tao Q, Sun Z, Xia L (2020) Correlation of chest CT and RT-PCR Testing in coronavirus disease 2019 (COVID-19) in China: a report of 1014 cases. Radiology 26:200642. https://doi.org/10.1148/radiol.2020200642

18. Fang Y, Zhang H, Xie J, Lin M, Ying L, Pang P, Ji W (2020) Sensitivity of chest CT for COVID-19: comparison to RT-PCR. Radiology. https://doi.org/10.1148/radiol.2020200432
19. Fields BKK, Demirjian NL, Gholamrezanezhad A (2020) Coronavirus disease 2019 (COVID-19) diagnostic technologies: a country-based retrospective analysis of screening and containment procedures during the first wave of the pandemic. Clin Imaging 67:219-225. https://doi.org/10.1016/j.clinimag.2020.08.014

20. Raptis CA, Hammer MM, Short RG, Shah A, Bhalla S, Bierhals AJ, Filev PD, Hope MD, Jeudy J, Kligerman SJ, Henry TS (2020) Chest CT and coronavirus disease (COVID-19): a critical review of the literature to date. AJR Am J Roentgenol 16:1-4. https://doi. org/10.2214/AJR.20.23202

21. Kooraki S, Hosseiny M, Myers L, Gholamrezanezhad A (2020) Coronavirus (COVID-19) outbreak: what the department of radiology should know. J Am Coll Radiol 17(4):447-451. https://doi. org/10.1016/j.jacr.2020.02.008

22. Hosseiny M, Kooraki S, Gholamrezanezhad A, Reddy S, Myers L (2020) Radiology perspective of coronavirus disease 2019 (COVID-19): lessons from severe acute respiratory syndrome and middle east respiratory syndrome. AJR Am J Roentgenol 214(5):1078-1082. https://doi.org/10.2214/AJR.20.22969

23. Shi H, Han X, Jiang N, Cao Y, Alwalid O, Gu J, Fan Y, Zheng C (2020) Radiological findings from 81 patients with COVID-19 pneumonia in Wuhan, China: a descriptive study. Lancet Infect Dis 20(4):425-434

24. Salehi S, Abedi A, Radmard AR, Sorouri M, Gholamrezanezhad A (2020) Chest computed tomography manifestation of coronavirus disease 2019 (COVID-19) in patients with cardiothoracic conditions. J Thorac Imaging. https://doi.org/10.1097/RTI.00000 00000000531

25. Ye Z, Zhang Y, Wang Y, Huang Z, Song B (2020) Chest CT manifestations of new coronavirus disease 2019 (COVID-19): a pictorial review. Eur Radiol 30(8):4381-4389

26. Salehi S, Abedi A, Gholamrezanezhad A (2020) Reply to "vascular changes detected with thoracic CT in coronavirus disease (COVID-19) might be significant determinants for accurate diagnosis and optimal patient management". AJR Am J Roentgenol. https://doi.org/10.2214/AJR.20.23339

27. Tanaka N, Kim JS, Newell JD, Brown KK, Cool CD, Meehan R, Emoto T, Matsumoto T, Lynch DA (2004) Rheumatoid arthritisrelated lung diseases: CT findings. Radiology 232(1):81-91. https ://doi.org/10.1148/radiol.2321030174

28. Cojocaru M, Cojocaru IM, Silosi I, Vrabie CD, Tanasescu R (2020) Extra-articular manifestations in rheumatoid arthritis. Maedica (Buchar) 5(4):286-291

29. Joob B, Wiwanitkit V (2020) SLE, hydroxychloroquine and no SLE patients with COVID-19: a comment. Ann Rheum Dis 79(6):e61. https://doi.org/10.1136/annrheumdis-2020-217506

30. Sawalha AH, Zhao M, Coit P, Lu Q (2020) Epigenetic dysregulation of ACE2 and interferon-regulated genes might suggest increased COVID-19 susceptibility and severity in lupus patients. Clin Immunol 215:108410. https://doi.org/10.1016/j. clim.2020.108410

31. Kamen DL, Strange C (2010) Pulmonary manifestations of systemic lupus erythematosus. Clin Chest Med 31(3):479-488. https ://doi.org/10.1016/j.ccm.2010.05.001

32. Li P, Li S, Li L, Wang M (2017) Chest CT findings in systemic lupus erythematosus and its correlation with serum markers. Radiol Infect Dis 4(1):7-13

33. Del Papa N, Sambataro G, Minniti A, Pignataro F, Caporali R (2020) Novel COronaVirus Disease 2019 (COVID-19) epidemic: what are the risks for systemic sclerosis patients? Autoimmun Rev. https://doi.org/10.1016/j.autrev.2020.102558

34. Matucci-Cerinic M, Bruni C, Allanore Y (2020) Systemic sclerosis and the COVID-19 pandemic: World Scleroderma Foundation preliminary advice for patient management. Ann Rheum Dis 79:724-726 
35. Cheng C, Li C, Zhao T, Yue J, Yang F, Yan Y, Liu X (2020) COVID-19 with rheumatic diseases: a report of 5 cases. Clin Rheumatol. https://doi.org/10.1007/s10067-020-05160-x

36. Mihai C, Dobrota R, Schröder M, Garaiman A, Jordan S, Becker MO, Maurer B, Distler O (2020) COVID-19 in a patient with systemic sclerosis treated with tocilizumab for SSc-ILD. Ann Rheum Dis 79(5):668-669. https://doi.org/10.1136/annrheumdi s-2020-217442

37. Strollo D, Goldin J (2010) Imaging lung disease in systemic sclerosis. Curr Rheumatol Rep 12(2):156-161. https://doi. org/10.1007/s11926-010-0095-0

38. Li K, Wu J, Wu F, Guo D, Chen L, Fang Z, Li C (2020) The clinical and chest $\mathrm{CT}$ features associated with severe and critical COVID-19 pneumonia. Invest Radiol 55(6):327-331. https://doi. org/10.1097/RLI.0000000000000672

39. Emad Y, Abdel-Razek N, Gheita T, El-Wakd M, El-Gohary T, Samadoni A (2007) Multislice CT pulmonary findings in Behçet's disease (report of 16 cases). Clin Rheumatol 26(6):879-884. https ://doi.org/10.1007/s10067-006-0408-x

40. Ceylan N, Bayraktaroglu S, Erturk SM, Savas R, Alper H (2010) Pulmonary and vascular manifestations of Behcet disease: imaging findings. AJR Am J Roentgenol 194(2):W158-W164. https:// doi.org/10.2214/AJR.09.2763

41. Tomelleri A, Sartorelli S, Campochiaro C, Baldissera EM, Dagna L (2020) Impact of COVID-19 pandemic on patients with largevessel vasculitis in Italy: a monocentric survey. Ann Rheum Dis. https://doi.org/10.1136/annrheumdis-2020-217600

42. Gianfrancesco MA, Hyrich KL, Gossec L, Strangfeld A, Carmona L, Mateus EF, Sufka P, Grainger R, Wallace Z, Bhana S, Sirotich E, Liew J, Hausmann JS, Costello W, Robinson P, Machado PM, Yazdany J (2020) COVID-19 Global Rheumatology Alliance
Steering Committee. Rheumatic disease and COVID-19: initial data from the COVID-19 Global Rheumatology Alliance provider registries. Lancet Rheumatol. https://doi.org/10.1016/S2665 -9913(20)30095-3

43. Martinez F, Chung JH, Digumarthy SR, Kanne JP, Abbott GF, Shepard JA, Mark EJ, Sharma A (2012) Common and uncommon manifestations of Wegener granulomatosis at chest CT: radiologic-pathologic correlation. Radiographics 32(1):51-69. https://doi.org/10.1148/rg.321115060

44. Cordier JF, Valeyre D, Guillevin L, Loire R, Brechot JM (1990) Pulmonary Wegener's granulomatosis. A clinical and imaging study of 77 cases. Chest 97(4):906-912. https://doi.org/10.1378/ chest.97.4.906

45. Wojciechowska J, Krajewski W, Krajewski P, Kręcicki T (2016) Granulomatosis with polyangiitis in otolaryngologist practice: a review of current knowledge. Clin Exp Otorhinolaryngol 9(1):813. https://doi.org/10.21053/ceo.2016.9.1.8

46. Salehi S, Reddy S, Gholamrezanezhad A (2020) Long-term pulmonary consequences of coronavirus disease 2019 (COVID19): what we know and what to expect. J Thorac Imaging 35(4):W87-W89

47. Katal S, Aghaghazvini L, Gholamrezanezhad A (2020) Chest-CT findings of COVID-19 in patients with pre-existing malignancies; a pictorial review. Clin Imaging 67:121-129. https://doi. org/10.1016/j.clinimag.2020.06.004

Publisher's Note Springer Nature remains neutral with regard to jurisdictional claims in published maps and institutional affiliations. 